\title{
Reabilitação oral de paciente com fissura labiopalatina: relato de caso
}

\author{
Oral rehabilitation of a patient with cleft lip and palate: a case report \\ Rehabilitación oral de un paciente con labio y paladar hendido: reporte de un caso
}

Recebido: 29/01/2022 | Revisado: 03/02/2022 | Aceito: 09/02/2022 | Publicado: 14/02/2022

Célia Patrícia Müller Rodrigues ORCID: https://orcid.org/0000-0002-7752-0560 Universidade Estadual do Oeste do Paraná, Brasil E-mail: pattymiiller@hotmail.com

Aline Vaneli Pelizzoni

ORCID: https://orcid.org/0000-0003-1125-0200

Universidade Estadual do Oeste do Paraná, Brasil E-mail: aline.vaneli@gmail.com

Jeani Aparecida Petrik Brischke

ORCID: https://orcid.org/0000-0002-6347-8242

Universidade Estadual do Oeste do Paraná, Brasil E-mail: jeanipetrik1@gmail.com

Scheila Kazmierski

ORCID: https://orcid.org/0000-0002-9902-8278 Pontifícia Universidade Católica do Paraná, Brasil

E-mail: contato@ scheilakazmierski.com.br

Mariângela Monteiro de Melo Baltazar

ORCID: https://orcid.org/0000-0001-6395-9516

Universidade Estadual do Oeste do Paraná, Brasil E-mail: mmmwgb@uol.com.br

Rejane Teixeira Coelho

ORCID: https://orcid.org/0000-0002-8630-9388 Universidade Estadual do Oeste do Paraná, Brasil E-mail: enajerco@uol.com.br

Daniela Pereira Lima

ORCID: https://orcid.org/0000-0002-9691-4880 Universidade Estadual do Oeste do Paraná, Brasil E-mail: dani.pl@hotmail.com

\begin{abstract}
Resumo
A fissura labiopalatina é a anomalia craniofacial mais comum em seres humanos (1:650 nascimentos), sendo sua etiologia multifatorial. Os pacientes com essa condição são acometidos por desordens morfológicas e funcionais, dentre elas, má formações dentárias sendo a mais comum a agenesia, que ocorre com maior frequência nos incisivos laterais superiores permanentes do lado onde está a fenda, afetando a função, a estética e consequentemente a autoestima do paciente. O objetivo desse trabalho foi relatar o caso de um paciente fissurado com agenesia dentária, apresentar a alternativa utilizada para sua reabilitação e o impacto psicológico do tratamento. O paciente foi atendido no CEAPAC (Centro de Atenção e Pesquisa em Anomalias Craniofaciais) sendo sua queixa principal a agenesia dos incisivos laterais superiores, a placa de acrílico com dentes de estoque utilizada para reabilitação e a estética dos incisivos centrais superiores que se encontravam desalinhados e com alteração de forma. O plano de tratamento proposto foi a confecção de duas próteses fixas, tipo cantilever, envolvendo os incisivos centrais e os caninos superiores como apoio para o elemento suspenso, já que houve rejeição do enxerto e a instalação de implantes era inviável. A reabilitação dentária foi concluída de forma satisfatória, devolvendo ao paciente, dentro de suas limitações, não somente a função, como também a estética e autoconfiança, o paciente continuará em acompanhado para proservação clínica do caso. Ressaltase a importância do atendimento por uma equipe multiprofissional especializada, considerando a complexidade da anomalia e a necessidade de reabilitação integral.
\end{abstract}

Palavras-chave: Agenesia dentária; Fenda labial; Prótese adesiva.

\begin{abstract}
Cleft lip and palate is the most common craniofacial anomaly in humans (1:650 births), and its etiology is multifactorial. Patients with this condition are affected by morphological and functional disorders, including dental malformations, the most common being agenesis, which occurs more frequently in the permanent maxillary lateral incisors on the side where the cleft is located, affecting function, aesthetics and consequently the patient's self-esteem. The objective of this study was to report the case of a cleft patient with tooth agenesis, to present the alternative used for his rehabilitation and the psychological impact of the treatment. The patient was treated at CEAPAC (Center for Attention and Research
\end{abstract}


in Craniofacial Anomalies) and his main complaint was the agenesis of the upper lateral incisors, the acrylic plate with stock teeth used for rehabilitation and the aesthetics of the upper central incisors that were misaligned and with shape change. The proposed treatment plan was the manufacture of two fixed prostheses, cantilever type, involving the central incisors and the maxillary canines as support for the suspended element, since there was rejection of the graft and the installation of implants was unfeasible. Dental rehabilitation was completed satisfactorily, returning to the patient, within its limitations, not only the function, but also the aesthetics and self-confidence, the patient will continue to be followed up for clinical follow-up of the case. The importance of care by a specialized multidisciplinary team is highlighted, considering the complexity of the anomaly and the need for comprehensive rehabilitation.

Keywords: Tooth agenesis; Cleft lip; Resin-bonded.

\section{Resumen}

El labio leporino y paladar hendido es la anomalía craneofacial más común en humanos (1:650 nacimientos), y su etiología es multifactorial. Los pacientes con esta condición se ven afectados por trastornos morfológicos y funcionales, entre ellos, malformaciones dentales, siendo la más común la agenesia, que se presenta con mayor frecuencia en los incisivos laterales permanentes maxilares del lado donde se encuentra la fisura, afectando la función, la estética y consecuentemente la salud del paciente. autoestima. El objetivo de este estudio fue reportar el caso de un paciente fisurado con agenesia dental, presentar la alternativa utilizada para su rehabilitación y el impacto psicológico del tratamiento. El paciente fue atendido en el CEAPAC (Centro de Atención e Investigación de Anomalías Craneofaciales) y su principal queja era la agenesia de los incisivos laterales superiores, la placa acrílica con dientes de serie utilizada para la rehabilitación y la estética de los incisivos centrales superiores desalineados y con cambio de forma. El plan de tratamiento propuesto fue la fabricación de dos prótesis fijas, tipo cantilever, involucrando los incisivos centrales y los caninos maxilares como soporte del elemento suspendido, ya que hubo rechazo del injerto y la instalación de implantes fue inviable. La rehabilitación dental se culminó satisfactoriamente, devolviéndole al paciente, dentro de sus limitaciones, no solo la función, sino también la estética y la confianza en sí mismo, el paciente continuará en seguimiento para seguimiento clínico del caso. Se destaca la importancia de la atención por un equipo multidisciplinario especializado, considerando la complejidad de la anomalía y la necesidad de rehabilitación integral.

Palabras clave: Agenesia dental; Labio leporino; Dentadura parcial fija con resina consolidada.

\section{Introdução}

A fissura labiopalatina, também chamada de fenda orofacial é a anomalia craniofacial mais comum em seres humanos, com prevalência nacional de 1:650 nascimentos. Sua etiologia é multifatorial e pode estar relacionada com fatores ambientais, genéticos e teratogênicos. Essa má formação ocorre entre a $4^{\circ}$ e $12^{a}$ semana do período gestacional, ocasionado pela falha na fusão dos processos embriológicos, podendo ser diagnosticada ainda nesse período a partir do exame de ultrassonografia (Costa et al.,2021; Schönardie et al.,2021).

O tratamento é complexo e longo, exigindo a atuação conjunta de diversas áreas da saúde, por afetar a função, estética e emocional dos pacientes fissurados (Teixeira et al., 2020). A severidade do caso varia de acordo com a extensão (completa, incompleta, unilateral ou bilateral) e localização da fissura, que de acordo com Spina podem ser classificadas como: Fissura labial pré forame incisivo, Fissura palatina pós forame incisivo e Fissura transforame, além das Fissuras raras de face (Alves, Moura \& Freitas, 2019).

Os pacientes acometidos por essa anomalia são submetidos a cirurgia corretiva do lábio (queiloplastia) a partir dos 3 meses de vida, e cirurgia corretiva de palato (palatoplastia) que ocorre normalmente aos 12 meses. Cirurgias secundárias podem ser necessárias de acordo com a gravidade do caso, bem como cirurgia de enxerto ósseo alveolar, por volta dos 10 anos e a cirurgia ortognática após o completo crescimento esquelético (Costa et al.,2020).

Esses pacientes também apresentam diversas desordens morfológicas e funcionais, dentre elas complicações na audição, fonação, sucção, deglutição, mastigação, respiração, psicossociais e também dentárias, como má oclusão, anomalias de forma e número, que contribuem para estética e funcionalidade deficientes (Costa et al., 2021). Estima-se que pacientes com fissuras tem maior possibilidade de apresentar anomalias dentárias em comparação a pacientes que não possuem fissura (Lavôr et al., 2020).

A má formação dentária mais comum em pacientes com fenda é a ausência congênita dos germes dentários, conhecida como agenesia, que ocorre com maior frequência em dentes permanentes, geralmente os incisivos laterais superiores do lado onde está a fenda, podendo afetar gravemente a função, a estética e consequentemente a autoestima do paciente (Pinheiro, 2020). 
O objetivo desse trabalho é apresentar o caso de um paciente fissurado com agenesia dentária e apresentar a alternativa utilizada para sua reabilitação.

\section{Metodologia}

O relato do caso foi realizado no Centro de Atenção e Pesquisa em Anomalias Craniofaciais (CEAPAC) anexo ao Hospital Universitário do Oeste do Paraná (HUOP) na cidade de Cascavel - PR, por meio de entrevista com o paciente, observações e intervenções diretas.

A entrevista psicológica foi composta por um atendimento, sem tempo limitado. $\mathrm{O}$ atendimento foi gravado e posteriormente transcrito e analisado em seu conteúdo (Minayo, 2011). Utilizou-se também da técnica do Desenho-estória (Trinca, 2013), adaptada, foram solicitados dois desenhos, um de tema livre e outro que representasse o tratamento odontológico.

$\mathrm{O}$ relato seguiu os preceitos éticos, sendo aprovado previamente pelo comitê de ética em pesquisa sob o parecer $\mathrm{n}^{\circ}$ 4.250.143 e com o consentimento do paciente o qual assinou o Termo de Consentimento Livre e esclarecido.

\section{Relato de Caso}

Paciente do sexo masculino, 20 anos de idade com diagnóstico de fissura transforame completa bilateral. Iniciou o tratamento no CEAPAC aos 14 anos, já havia passado por cirurgia de queiloplastia primária e secundária em outro centro, bem como por cirurgia de palatoplastia e enxerto ósseo na região alveolar.

A queixa principal do paciente era a agenesia dos incisivos laterais superiores e a placa de acrílico com dentes de estoque utilizada para reabilitação, conforme é possível visualizar na figura 1A, ele ainda relatou insatisfação em relação estética dos incisivos centrais superiores que se encontravam desalinhados e com alteração de forma. Figura 1B

Ao exame clínico foi observada rarefação óssea na região da fissura, confirmada por radiografia panorâmica (Figura 1C) e tomografia computadorizada (Figura 1D), sendo possível constatar que houve rejeição do enxerto ósseo alógeno realizado na região aos 13 anos, inviabilizando a instalação de implantes para reabilitação. Também foi constatado no exame clínico que não haviam lesões cariosas ou inflamação gengival, estando todos os dentes hígidos. 
Figura 1. A - Placa de acrílico com dentes de estoque utilizada pelo paciente antes do tratamento. B - Alteração de forma dos incisivos centrais e regiões acometidas pela agenesia. C e D - Imagem panorâmica e TC.

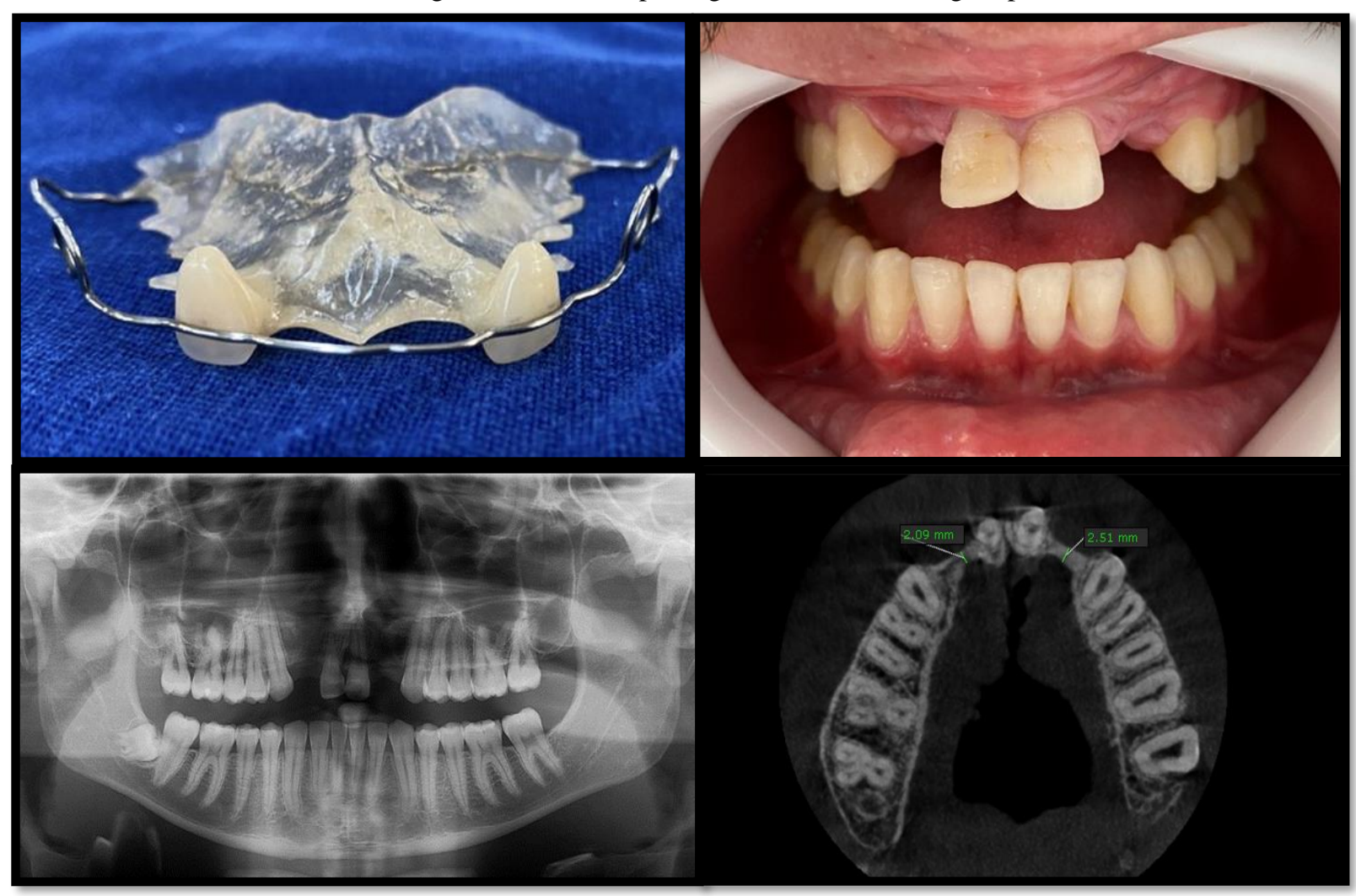

Fonte: Autores.

O plano de tratamento proposto foi a confecção de duas próteses fixas, tipo cantilever, envolvendo os incisivos centrais e os caninos superiores como apoio para o elemento suspenso. Sob o consentimento do paciente foi iniciado o procedimento, realizando a moldagem para pedido das próteses provisórias, uma do dente 13 ao 11 e outra do dente 23 ao 21 .

Com as próteses em mãos, os dentes foram preparados sob anestesia local, pois se apresentavam vitais (Figura 2A). Nova moldagem com silicona de adição foi realizada, para pedido dos copings, e as provisórias foram cimentadas (Figura 2B). 
Figura 2. A - Preparo dos incisivos centrais e caninos. B - Próteses provisórias cimentadas. C - Copings em resina. D - Próteses em zircônia com incisivo lateral em cantilever cimentadas.

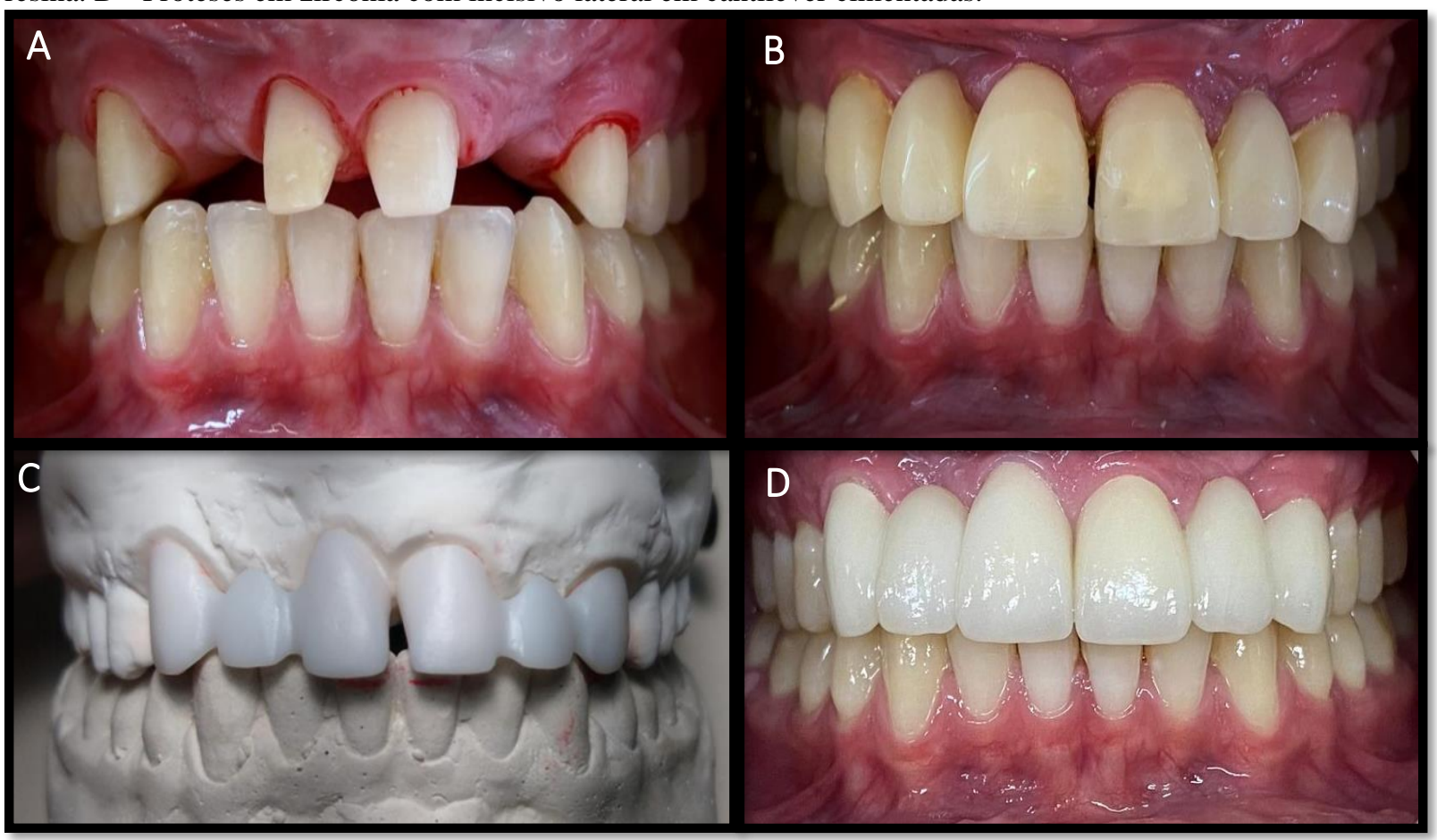

Fonte: Autores.

Após a prova dos copins em resina (Figura 2C), foram solicitadas as peças em Zircônia. Em conversa com o paciente foi selecionada a cor B1 e o mesmo irá realizar clareamento dental posteriormente. As peças em Zircônia foram provadas e cimentadas com Cimento de Zinco e os ajustes oclusais foram realizados (Figura 2D).

Durante todo o processo o paciente não relatou sensibilidade sendo que o resultado do trabalho atingiu sua expectativa e o mesmo retornará periodicamente para acompanhamento.

\section{Resultados e Discussão}

\section{Aspectos odontológicos}

Crianças portadoras de fissuras são submetidas a cirurgias corretivas ainda nos primeiros meses de vida, tais procedimentos resultam em restrição de crescimento maxilar, alteração de crescimento da face e consequentemente em má oclusão dentária (Martins et al.,2021). O paciente do relato, apesar de não ter grandes alterações no perfil facial, necessitou de tratamento ortodôntico para correção da oclusão.

Além das alterações descritas, a região da fissura ainda é acometida por alterações dentárias de forma e número (Martins et al., 2021). A frequência de agenesia dos incisivos laterais superiores varia de $42 \%$ a $64 \%$ em pacientes fissurados, dependendo da extensão e gravidade da fenda (Tomazoni et al., 2017 e Martins et al., 2021). Também é comumente encontrado nesses pacientes erupção ectópica e defeitos de esmalte (Lavôr et al., 2020). No caso relatado o paciente apresentava fissura bilateral, sendo que os dois lados foram afetados pela ausência do germe dental e os incisivos centrais por alterações de esmalte.

Essas alterações são descritas como decorrentes de falta de suplemento sanguíneo para as células mesenquimais da região devido a rarefação óssea, contudo intervenções cirúrgicas realizadas ainda no momento da formação do germe dental vêm sendo apontadas como responsáveis por deformações, e até extração do mesmo. (Cavalcante, 2020. Uchino et al., 2020). 
Apesar de todas essas variações, para cada caso há um tratamento reabilitador, sendo necessário avaliar algumas características clínicas, como perfil facial, tipo de oclusão, presença de apinhamento nas arcas e idade do paciente. (Silveira et al., 2016).

O tratamento reabilitador mais comumente encontrado para pacientes que apresentam agenesia é a instalação de implantes osseointegrados e subsequente prótese sobre implante (Kiliaridis et al., 2016), contudo, conforme descrito, a rarefação óssea e a rejeição do enxerto contra-indicavam esse tratamento para o paciente do relato.

A segunda alternativa frequentemente encontrada é a reabilitação através de próteses adesivas, nas quais o incisivo lateral superior atua como cantilever (Kiliaridis et al., 2016). No caso descrito optou-se por realizar as duas próteses cantilever, com coras totais cimentadas nos incisivos centrais e caninos devido a queixa estética do paciente. Se tratando de casos estéticos, em dentes vitais anteriores o tratamento recomendado é a confecção de coroas metal free em zircônia, utilizada no caso descrito, ou discilicato de lítio (Nomoto et al.,2021).

A literatura aponta que essas próteses apresentam excelentes resultados, em relação a estética, saúde do periodonto, higiene facilitada, retenção mais confortável para o paciente e maior longevidade, quando comparadas com próteses parciais removíveis (Tavares et al., 2020).

Os dentes pilares utilizados nesse caso apresentavam-se hígidos, com boa saúde periodontal, raízes longas e coroa clínica com estrutura para dar retenção aos cantilever, além disso, a oclusão encontrava-se estável conforme é recomendado por Tavares et al. 2020.

Outra opção de tratamento é o fechamento ortodôntico do espaço pertencente ao incisivo lateral superior, e posterior reanatomização dos caninos (Kiliaridis et al., 2016), contudo devido aos aspectos clínicos, essa não era uma opção viável para o caso relatado.

\section{Aspectos psicológicos}

Independente de qual tratamento é realizado, todos buscam amenizar os impactos causados pelo estigma da fissura, pois grande parte desses pacientes apresentam insatisfação com a sua estética, dentição, fala, entre outros fatores que causam desajuste psicossocial (Leite, 2020).

Nesse sentido, a entrevista psicológica evidenciou o impacto no autoconceito do paciente sobre as questões estéticas, funcionais, afetivas e em suas relações sociais. Autoconceito é entendido aqui como o modo que se pensa e sente a respeito de si e que tem influência direta na auto-estima, que por sua vez, diz respeito aos sentimentos de realização, amor próprio e mais valia (Graciano et al.,2007)

Na avaliação do paciente, houve diferença significativa no modo que se relacionava socialmente, desde a utilização da prótese provisória. Após a implantação do material definitivo teve expressiva melhora na sua qualidade de vida, que para ele era inimaginável, como pode ser observada na seguinte fala: "o que eu tenho hoje é mais do que eu sonhava antes, mais do que o resultado que eu queria". Aspectos como a timidez e insegurança nas relações, tanto sociais como afetivas, tiveram ganhos qualitativos que impactam, inclusive na sua autoestima "eu tinha uma visão de que eu preferia não trabalhar com pessoas, preferia não estar no meio de pessoas e ser mais reservado [...], porém, hoje eu prefiro mil vezes trabalhar com pessoas, prefiro mil vezes estar com pessoas, prefiro mil vezes dialogar, conversar, totalmente diferente por conta do ocorrido [...].

A transformação produzida pela prótese dentária, trouxe benefícios para o paciente que extrapolam o campo funcional e estético, a partir disso o autorizaram a se colocar em um outro lugar social, reconhecendo e se apropriando de sua identidade, quer dizer, da sua condição de sujeito para além da anomalia e da marca física causada por ela. O procedimento possibilitou uma autoidentificação das suas habilidades e características. 
O desenho-estória pode traduzir o impacto emocional da anomalia e do tratamento reabilitador. Foi proposto ao paciente que fizesse um desenho livre (Figura 3). Seu primeiro desenho foi o coração, o qual representou para o paciente a dificuldade de formação de vínculos afetivos devido à fissura. Ainda parte do desenho livre, a cruz, foi representada como símbolo de sua "fé" e "gratidão" aos seus familiares que o acompanharam em todo o processo de reabilitação. De um lado, expressa a exclusão social pela diferença e, de outro, os recursos emocionais utilizados para fortalecimento e enfrentamento. No inquérito feito sobre o desenho o nome atribuído a primeira representação foi "Extinção”.

Figura 3. Desenho livre - Nome: "Extinção".

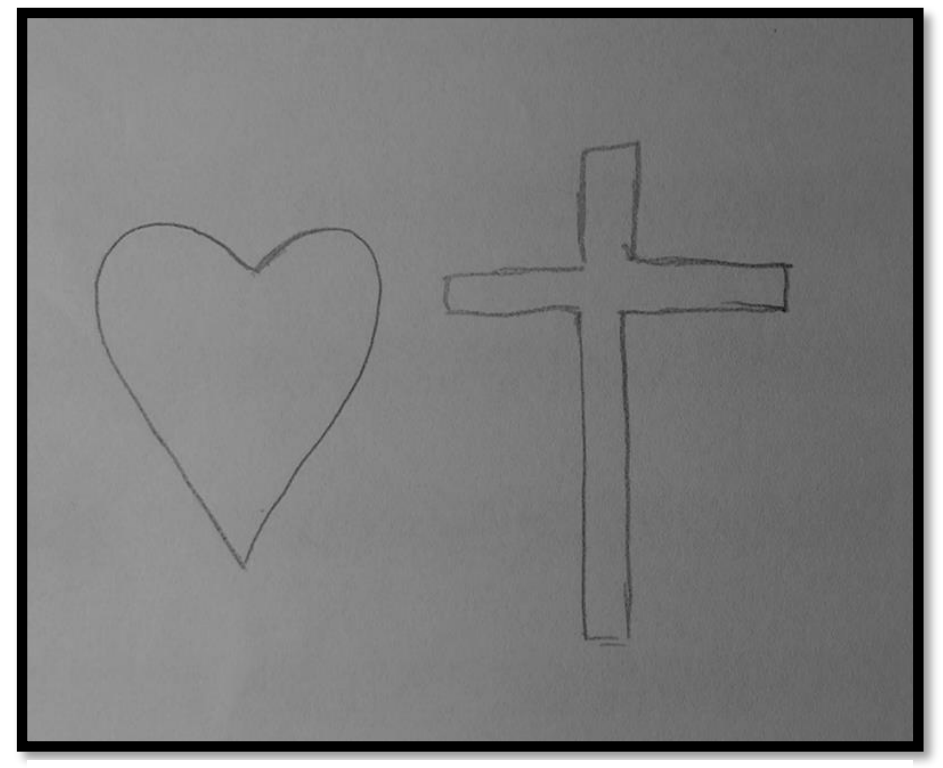

Fonte: Autores.

O segundo desenho proposto (Figura 4), foi solicitado com o tema: o tratamento. A imagem do avião como símbolo do tratamento, foi exposto na estória como algo que "leva para cima", "ao topo", quer dizer, que elevou sua autoestima e fé. O vôo do avião sob as nuvens, representou "a aceitação da condição da fissura e do modo que as relações se davam". A comparação entre o avião e o tratamento com a frase: "feitos por mãos humanas", demonstrou a importância e a dimensão obtida pelo tratamento. O título atribuído ao desenho foi: “Superação", associada à sua fé. 
Figura 4. Desenho temático - Nome: "Superação".

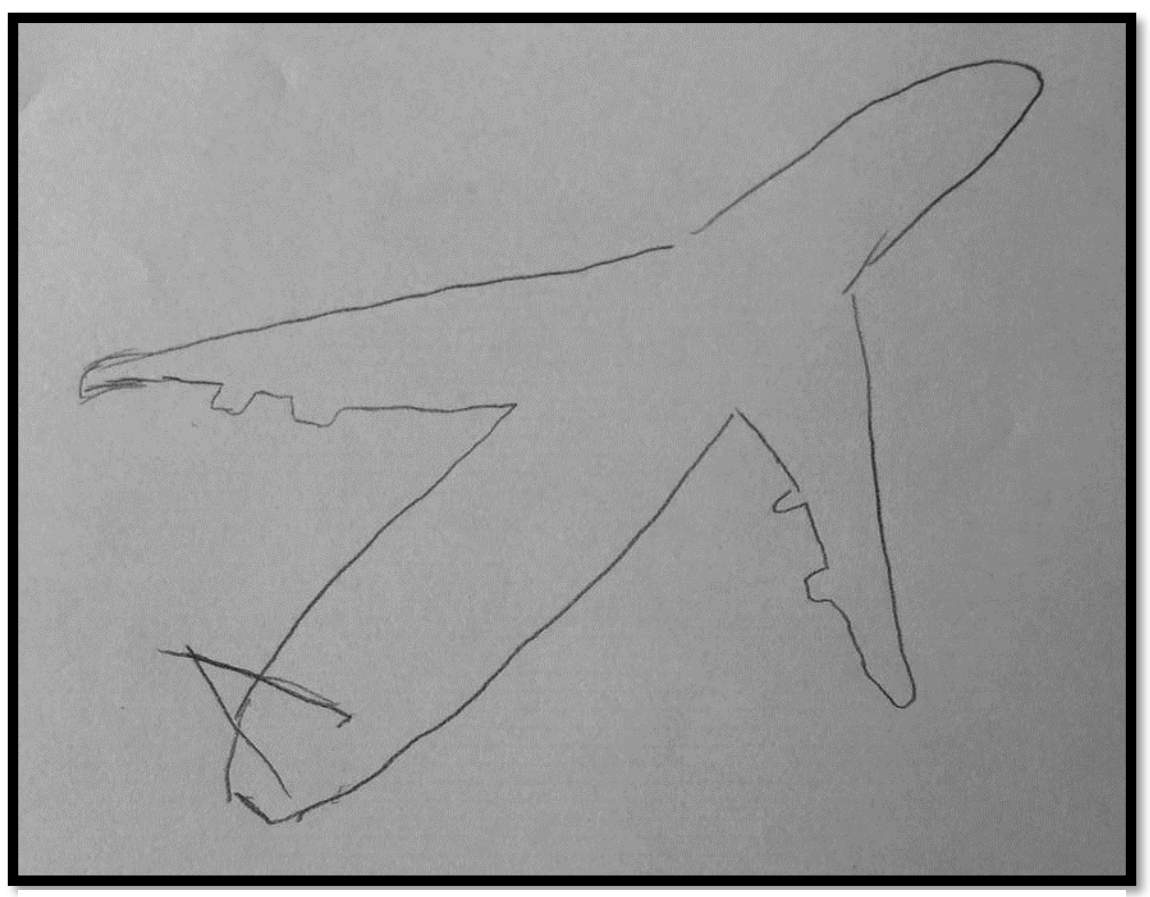

Fonte: Autores.

É importante destacar que o paciente não estava em tratamento psicológico, portanto a transformação, não só física, mas psicoafetiva do paciente foi possível pela delicadeza do trabalho clínico, efetivo e humanizado, proposto pelo serviço de odontologia, associado a reserva de recursos psíquicos próprios do paciente que se propôs ativo durante todo o tratamento. Contudo, cabe destacar, que o trabalho do psicólogo é fundamental para dar suporte, dentre tantas questões, na da autoimagem, pois o acometimento facial compromete a estética, um dos principais aspectos da socialização, tendo implicações na saúde mental (Paiva et al., 2020).

\section{Considerações Finais}

Para uma completa reabilitação é importante que a criança com fissura labiopalatina seja precocemente encaminhada para avaliação e tratamento, sendo acompanhada, a partir de então, por uma equipe multidisciplinar capaz de dar assistência integral ao indivíduo (Andrade, Rodrigues \& Santos, 2019). O paciente será acompanhado para proservação clínica do caso.

A reabilitação dentária descrita foi concluída de forma satisfatória, devolvendo ao paciente, dentro de suas limitações, não somente a função, como também a estética. Ao encontro disso, a entrevista psicológica e descrição do Desenho-Estória puderam evidenciar a autopercepção do paciente sobre o tratamento, em relação a sua adaptação e realização social, que são aspectos essenciais para considerar o tratamento como reabilitador, com possibilidade de transformação no modo de vida e na sua qualidade.

Ressalta-se a importância do atendimento por uma equipe multiprofissional especializada, considerando a complexidade da anomalia e a necessidade de reabilitação integral do paciente. A literatura ainda é escassa sobre o atendimento odontológico a pacientes com fissura labiopalatina, sendo necessário que novos trabalhos sejam elaborados a fim de contribuir para o conhecimento da anomalia e seus tratamentos. 


\section{Referências}

Alves, L. D. B., Moura, J. R., \& Freitas, V. S. (2019) Análise epidemiológica das fissuras labiais e/ou palatinas no município de Feira de Santana: estudo de corte transversal. J Dent Pub H., 10(1). https://doi.org/10.17267/2596-3368dentistry.v10i1.2244

Amaral, V. L. A. R. D. (1996). Aspectos psicológicos, problemas sociais e familiares associados às fissuras labiopalatinas. In Tratamento das fissuras labiopalatinas. 19-24

Andrade, C. A., Rodrigues, M. C., \& Santos, W. L. (2019). A Importância da Equipe Multiprofissional para a recuperação da criança com fenda labiopalatina. Revista Enfermagem Atual In Derme, 90(28). https://doi.org/10.31011/reaid-2019-v.90-n.28-art.512

Cavalcante, B. G. N. (2020). Alterações de desenvolvimento dentário em pacientes nascidos com fissuras labiopalatinas. Dissertação de Mestrado, Universidade Estadual da Paraíba, João Pessoa, PB, Brasil. https://repositorio.ufpb.br/jspui/bitstream/123456789/18462/1/BiancaGolzioNavarroCavalcante_Dissert.pdf

Costa, L. H., Amaral, B. P. A. de, \& Silva, J. P. P. (2021). Fissura labiopalatina: revisão literária. Revista saúde multidisciplinar, 9(1). http://revistas.famp.edu.br/revistasaudemultidisciplinar/article/view/172

Costa, N. F., Borges, A. L. L., \& Almeida, S. A. de, (2020). Fissuras palatinas, inovações e novos meios de tratamento: um estudo introdutório. Facit Business and Technology Journal, 1(14). http://revistas.faculdadefacit.edu.br/index.php/JNT/article/view/530

Fialho, A., \& Jorge, K. O. (2017). Tratamento odontológico em crianças com fissura labiopalatal: revisão de literatura. Revista Interação, 19(2), 105-119. https://doi.org/10.33836/interacao.v19i2.140

Graciano, M. I. G., Tavano, L. D. A., \& Bachega, M. I. (2007). Aspectos psicossociais da reabilitação. In Fissuras labiopalatinas: uma abordagem interdisciplinar. 311-333.

Kiliaridis, S., Sidira, M., Kirmanidou, Y., \& Michalakis, K. (2016). Treatment options for congenitally missing lateral incisors. Eur J Oral Implantol, 9(Suppl 1), S5-24. https://pubmed.ncbi.nlm.nih.gov/27314109/

Lavôr, J. R., Lacerda, R. H. W., Modesto, A., \& Vieira, A. R. (2020). Maxillary incisor enamel defects in individuals born with cleft lip/palate. Plos one, 15(12), e0244506. https://doi.org/10.1371/journal.pone.0244506

Leite, R. B. (2020). Fissura labiopalatina: estudo do papel do profissional de saúde na diminuição dos danos ao paciente. Revista Ciências e Odontologia, 4(1), 48-55. http://revistas.icesp.br/index.php/RCO/article/view/707

Martins, J. R., Teixeira, S. M., Oliveira, I. M. de, Carneiro, G. K. M., \& Favretto, C. O. (2021). Desenvolvimento da dentição decídua em paciente com fissura labiopalatina bilateral completa: relato de caso. Archives of Health Investigation, 10(2), 287-291. https://doi.org/10.21270/archi.v10i2.4878

Minayo, M. C. de S., Deslandes, S. F., \& Gomes, R. (2011). Pesquisa social: teoria, método e criatividade. Editora Vozes Limitada.

Moreira, F. A. (2017). Agenesia dos incisivos laterais superiores: prevalência, diagnóstico e tratamento. Dissertação de Doutorado, Universidade Fernando Pessoa, Porto, Portugal. https://bdigital.ufp.pt/bitstream/10284/6202/1/PPG_35130.pdf

Moura Barros, E. F. de, Moura, W. da S., Henriques, J. F. C., Ferreira, J. de A., \& Martins, G. A. S. (2018). Prevalence of diastema between upper central incisors upper in patients with agenesis of the lateral maxillary incisor. Focus Oral Research, 1(1), 44-52. https://doi.org/10.35169/for.v1i1.11

Nomoto, S., Sugiuchi, A., Asai, T., Sato, T., \& Sekine, H. (2021). Patient with Poor Esthetic Anterior Tooth Position Treated with Lithium Disilicate Mesial Cantilever Single-retainer Fixed Partial Denture: A Case Report. The Bulletin of Tokyo Dental College, 62(2), 119-125. https://doi.org/10.2209/tdcpublication.2020-0038

Paiva, I. T., Lemos, D. I. M., Alves, H., \& Avoglia, H. R. C., (2020). Sentindo-se diferente: uma revisão bibliográfica sobre a autoestima da pessoa com fissura labial e/ou palatina. PsicolArgum, 38(101),580-603. http://dx.doi.org/10.7213/psicolargum.38.101.AO09

Pinheiro, T. (2020). Condições bucais em pacientes com fissuras lábio palatais: revisão de literatura. Trabalho de Conclusão de Curso. Centro Universitário Uniguairacá, Guarapuava, PR, Brasil. http://200.150.122.211:8080/jspui/handle/23102004/247

Rocha, C. L. (2018). Perfil de saúde bucal do paciente portador de fissura labiopalatina: estudo epidemiológico em hospital pediátrico do Nordeste Brasileiro. Trabalho de Conclusão de Curso, Universidade Federal do Ceará, Fortaleza, CE, Brasil. http://www.repositorio.ufc.br/handle/riufc/34837

Sá, J. O., Maranhão, S. C., Canguçú, D. L., Coutinho, T. S. L., Medrado, A. P., \& Reis, S. R. A. (2014). Anomalias Dentárias nas fissuras labiais e/ou palatinas não-sindrômicas: revisão da literatura. Revista Bahiana de Odontologia [Internet], 153-159. https://dx.doi.org/10.17267/2238-2720revbahianaodonto.v4i3.495

Schönardie, M. S., Ribas, L. P., Wagner, G. P., \& Cardoso, M. C. D. A. F. (2021). Relação entre o desenvolvimento infantil e as fissuras labiopalatinas. Distúrbios da Comunicação, 33(1), 40-48. https://doi.org/10.23925/2176-2724.2021v33i1p40-48

Schwartz, J. P., Somensi, D. S., Yoshizaki, P., Reis, L. L. S., Lauris, R. D. C. M. C., Silva, O. G. D. \& Garib, D. G. (2014). Prevalence of dental anomalies of number in different subphenotypes of isolated cleft palate. Dental press journal of orthodontics, 19, 55-59. https://doi.org/10.1590/2176-9451.19.1.055-059.oar

Silva, F. M. D., Carvalho, P. M. D., \& Côrtes, L. C. A. (2020). Agenesia dentária em adolescente: uma abordagem multidisciplinar. Relato de caso. (UNIT-SE. Trabalho de Conclusão de Curso, Universidade de Tiradentes, Aracaju, SE, Brasil. http://openrit.grupotiradentes.com:8080/xmlui/handle/set/3550

Silveira, G. S., Almeida, N. V. de, Pereira, D. M. T., Mattos, C. T., \& Mucha, J. N. (2016). Prosthetic replacement vs space closure for maxillary lateral incisor agenesis: a systematic review. American Journal of Orthodontics and Dentofacial Orthopedics, 150(2), 228-237. https://doi.org/10.1016/j.ajodo.2016.01.018 
Research, Society and Development, v. 11, n. 3, e10511326306, 2022

(CC BY 4.0) | ISSN 2525-3409 | DOI: http://dx.doi.org/10.33448/rsd-v11i3.26306

Souza, R. M. de, Oliveira, H. T. de, \& Farret, M. M. (2020). Tratamento ortodôntico-cirúrgico de fissura labiopalatina unilateral associada a mordida cruzada total e agenesia de incisivo lateral e pré-molar superiores: relato de caso. Revista Clínica de Ortodontia Dental Press, 19(1). https://br.clinicalorthodontics.net/rcodp-v19n01-2020-58/

Tavares, J. S., Klautau, E. B., Maranhão, K., \& Reis, A. C. D. S. (2020). Prótese Parcial Fixa em Cantilever, uma opção de tratamento: Revisão da Literatura. Rev. Salusvita (Online), 141-151. https://pesquisa.bvsalud.org/portal/resource/pt/biblio-1140266

Teixeira, A. C. D. S., \& Barros, M. F. M. D. (2020). Proposta de protocolo de tratamento de pacientes com fissura labiopalatina baseado em mínima intervenção. Trabalho de Conclusão de Curso, Centro Universitário FAMETRO - UNIFAMETRO, Fortaleza, CE, Brasil. http://repositorio.fametro.com.br/jspui/handle/123456789/741

Tomazoni, F., Bertelli, A. E. (2017, Outubro). Prevalência de anomalias dentárias em pacientes com fissuras labiais e palatinas e fatores associados: um estudo transversal. Anais do V Congresso de Pesquisa e Extensão da Faculdade da Serra Gaúcha, 5(5), Caxias do Sul, RS, Brasil. http://tede.unioeste.br/handle/tede/3708

Trinca, W. (2013). Formas compreensivas de investigação psicológica: procedimento de desenhos-estórias e procedimentos de desenhos de família com estórias. São Paulo: Vetor.

Uchino, M., Arima, H., Kobayashi, H., Nagata, J., Yasumura, T., Ishii, T.\& Sueishi, K. (2020). Survey on Condition and Orthodontic Treatment of Maxillary Lateral Incisors in Patients with Cleft Lip and Palate. The Bulletin of Tokyo Dental College, 61(2), 95-102. https://doi.org/10.2209/tdcpublication.2019-0013 